\title{
WEAK STABILITY OF INTERVAL ORBITS OF CIRCULANT MATRICES IN FUZZY ALGEBRA
}

\author{
Helena MYŠKOVÁ \\ Department of Mathematics and Theoretical Informatics, Faculty of Electrical Engineering and Informatics, Technical University of \\ Košice, Němcovej 32, 04200 Košice, Slovak Republic, tel.: +421 55602 2445, e-mail: helena.myskova@tuke.sk
}

\begin{abstract}
Fuzzy algebra is an algebraic structure in which classical addition and multiplication are replaced by $\oplus$ and $\otimes$, where $a \oplus b=$ $\max \{a, b\}, a \otimes b=\min \{a, b\}$. An orbit of $A$ generated by $x$ is called stable if $\operatorname{per}(A, x)=1$. An interval orbit of an interval matrix $\mathbf{A}$ and an interval vector $\mathbf{X}$ and the weak stability of an interval orbit are defined. A necessary and sufficient condition for the weak stability of interval orbits of circulant matrices is introduced and justified.
\end{abstract}

Keywords: fuzzy algebra, interval matrix, interval vector, interval orbit, stable orbit, the weak stability of an interval orbit

\section{INTRODUCTION}

Matrices in fuzzy algebra are useful for expressing applications of fuzzy discrete dynamic systems, graph theory, scheduling, knowledge engineering, cluster analysis, fuzzy systems and for describing diagnosis of technical devices [18], [19], medical diagnosis [15], [16] or fuzzy logic programs [10]. The problem studied in [15] leads to the problem of finding the greatest invariants of the fuzzy system (the greatest eigenvector of the fuzzy matrix corresponding to the greatest eigenvalue).

In practice, matrix and vector inputs are rather contained in some intervals than exact values. Considering matrices and vectors with interval coefficients is therefore of great practical importance, see [2], [8], [14]. The aim of this paper is to describe matrices and vectors with inexact data (interval matrices and vectors) for which there exists a stable orbit, i.e., an orbit with period equal to one, for some matrix and some vector from the given interval vector and interval matrix. The main result is concentrated in Theorem 5.1 which gives a necessary and sufficient condition for the weak stability of an interval orbit of circulant matrix which can be checked in $O\left(n^{2} \log n\right)$ arithmetic operations.

\section{PRELIMINARIES}

The fuzzy algebra $\mathscr{B}$ is the triple $(B, \oplus, \otimes)$, where $(B, \leq)$ is a bounded linearly ordered set with binary operations maximum and minimum, denoted by $\oplus$ and $\otimes$, respectively. The least element in $B$ will be denoted by $O$, the greatest one by $I$.

By $\mathbb{N}$ we denote the set of all natural numbers and by $\mathbb{N}_{0}$ the set $\mathbb{N}_{0}=\mathbb{N} \cup\{0\}$. The greatest common divisor of a set $S \subseteq \mathbb{N}$ is denoted by $\operatorname{gcd} S$ and the least common multiple by $\operatorname{lcm} S$. For a given natural number $n \in \mathbb{N}$, we use the notations $N=\{1,2, \ldots, n\}$ and $N_{0}=\{0,1, \ldots, n-1\}$.

For any $n \in \mathbb{N}, B(n, n)$ denotes the set of all square matrices of order $n$ and $B(n)$ the set of all $n$-dimensional column vectors over $\mathscr{B}$. The matrix operations over $\mathscr{B}$ are defined formally in the same manner (with respect to $\oplus, \otimes$ ) as matrix operations over any field. The $r$-th power of a matrix $A$ is denoted by $A^{r}$, with elements $\left(A^{r}\right)_{i j}$.

For $A \in B(n, n), C \in B(n, n)$ we write $A \leq C$ if $a_{i j} \leq c_{i j}$ holds true for all $i, j \in N$ and for $x, y \in B(n)$ we write $x \leq y$ if $x_{i} \leq y_{i}$ for each $i \in N$.

By digraph we understand a pair $\mathscr{G}=\left(V_{\mathscr{G}}, E_{\mathscr{G}}\right)$, where $V_{\mathscr{G}}$ is a non-empty finite set, called the node set, and $E_{\mathscr{G}} \subseteq V_{\mathscr{G}} \times V_{\mathscr{G}}$, called the arc set. A digraph $\mathscr{G}^{\prime}$ is a subdigraph of digraph $\mathscr{G}$, if $V_{\mathscr{G},} \subseteq V_{\mathscr{G}}$ and $E_{\mathscr{G}^{\prime}} \subseteq E_{\mathscr{G}}$. A path in $\mathscr{G}$ is the sequence of nodes $\mathscr{P}=\left(v_{0}, v_{1}, \ldots, v_{l}\right)$ such that $\left(v_{k-1}, v_{k}\right) \in E_{\mathscr{G}}$ for all $k=1,2, \ldots, l$. The number $l \geq 0$ is called the length of $\mathscr{P}$. If $v_{0}=v_{l}$, then $\mathscr{P}$ is called a cycle. A cycle is elementary if all nodes except the terminal node are distinct. A digraph is called strongly connected if any two distinct nodes of $\mathscr{G}$ are contained in a common cycle. By a strongly connected component of $\mathscr{G}$ we mean a maximal strongly connected subdigraph of $\mathscr{G}$. A strongly connected component $\mathscr{K}=\left(V_{\mathscr{K}}, E_{\mathscr{K}}\right)$ is called non-trivial if there is a cycle of positive length in $\mathscr{K}$. The strongly connected component of $\mathscr{G}$ containing node $i$ will be denoted by $\mathscr{G}[i]$. By $\mathrm{SCC}^{\star} \mathscr{G}$ we denote the set of all non-trivial components of $\mathscr{G}$.

\section{ORBIT PERIODICITY}

The notions of an orbit of $A$ generated by $x$ and known properties of the orbit periodicity are introduced in this section. A necessary and sufficient condition for the orbit period to be equal to one is proved.

Definition 3.1. For any $A \in B(n, n)$ and $x \in B(n)$ the orbit of $A$ generated by $x$ is the vector sequence $\mathscr{O}(A, x)=$ $\left(x(r) ; r \in \mathbb{N}_{0}\right)$ whose initial vector is $x(0)=x$ and successive members are defined by the formula $x(r+1)=A \otimes$ $x(r)$. The i-th coordinate of $x(r)$ is denoted by $x_{i}(r)$. The $i$-th coordinate orbit is the sequence $\mathscr{O}_{i}(A, x)=\left(x_{i}(r) ; r \in \mathbb{N}_{0}\right)$.

Definition 3.2. The sequence $S=(S(r) ; r \in \mathbb{N})$ is ultimately periodic if there is a natural number $p$ such that the following holds for some natural number $R$ :

$$
S(k+p)=S(k) \text { for all } k \geq R .
$$

The smallest natural number $p$ with the above property is called the period of $S$, denoted by $\operatorname{per}(S)$. The smallest $R$ with the above property is called the defect of $S$, denoted by $\operatorname{def}(S)$.

Both operations in fuzzy algebra are idempotent, so no new elements are created in the process of exponentiation of a 
matrix. Therefore any power sequence $\left(A^{k} ; k \in \mathbb{N}\right)$ contains only finite number of different matrices. The same holds true for any orbit. So a power sequence, an orbit $\mathscr{O}(A, x)$ and a coordinate orbit $\mathscr{O}_{i}(A, x)$ are always ultimately periodic sequences. Their periods will be called the period of $A$, the orbit period and the coordinate-orbit period, in notation $\operatorname{per}(A), \operatorname{per}(A, x)$ and $\operatorname{per}(A, x, i)$. Analogous notations $\operatorname{def}(A), \operatorname{def}(A, x)$ and $\operatorname{def}(A, x, i)$ will be used for the defects.

Theorem 3.1. 4, 17 Let $A \in B(n, n)$ and $x \in B(n)$. Then

$$
\begin{aligned}
& \text { i) } \operatorname{per}(A)=\operatorname{lcm}_{x \in B(n)} \operatorname{per}(A, x), \operatorname{def}(A)=\max _{x \in B(n)} \operatorname{def}(A, x), \\
& \text { ii) } \operatorname{per}(A, x)=\operatorname{lcm}_{i \in N} \operatorname{per}(A, x, i), \operatorname{def}(A, x)=\max _{i \in N} \operatorname{def}(A, x, i) \text {, } \\
& \text { iii) } \operatorname{per}(A, x(r))=\operatorname{per}(A, x) \text { for } r \in \mathbb{N}, \operatorname{def}(A, x(r))=0 \\
& \quad \text { for } r \geq \operatorname{def}(A, x) \text {. }
\end{aligned}
$$

Corollary 3.1. Let $A \in B(n, n)$ and $x \in B(n)$. $\operatorname{per}(A, x) \mid \operatorname{per}(A)$ for each $x \in B(n)$.

A matrix (vector) is called binary if $a_{i j} \in\{O, I\} \quad\left(x_{j} \in\right.$ $\{O, I\})$ for each $i, j \in N$.

Definition 3.3. Let $A \in B(n, n)$ be a binary matrix and $x \in B(n)$ be a binary vector. Then by $\mathscr{G}(A)$ we understand the digraph $\left(V_{\mathscr{G}_{(A)}}, E_{\mathscr{G}_{(A)}}\right)$ with $V_{\mathscr{G}_{(A)}}=\left(N, E_{\mathscr{G}(A)}\right)=$ $\left\{(i, j) ; a_{i j}=I\right\}$ and by $\mathscr{G}(A, x)$ we understand the corresponding node-weighted digraph obtained from $\mathscr{G}(A)$ by appending weight $x_{i}$ to each node $i$. A path in $\mathscr{G}(A, x)$ is called an orbit path if the weight of its terminal node is $I$.

Remark 3.1. We shall say that two strongly connected components $\mathscr{K}_{1} \in \mathrm{SCC}^{\star} \mathscr{G}(A, x)$ and $\mathscr{K}_{2} \in \operatorname{SCC}^{\star} \mathscr{G}(B, y)$ are identical, in notation $\mathscr{K}_{1}=\mathscr{K}_{2}$, if $V_{\mathscr{K}_{1}}=V_{\mathscr{K}_{2}}$ and $E_{\mathscr{K}_{1}}=E_{\mathscr{K}_{2}}$, i.e., the evaluation of vertices is not relevant.

Definition 3.4. For $A \in B(m, n)$ and $h \in B$, the threshold matrix $A_{(h)}$ corresponding to the threshold $h$ is a binary matrix of the same type as A, defined as follows:

$\left(A_{(h)}\right)_{i j}= \begin{cases}I & \text { if } a_{i j} \geq h \\ O & \text { otherwise }\end{cases}$

The associated digraphs $\mathscr{G}\left(A_{(h)}\right)$ and $\mathscr{G}\left(A_{(h)}, x_{(h)}\right)$ will be called the threshold digraphs corresponding to the threshold h.

Since any vector is viewed as an $(n \times 1)$ matrix, the above definition concerns also vectors.

For $A \in B(n, n)$ and $x \in B(n)$, the threshold orbit $\mathscr{O}\left(A_{(h)}, x_{(h)}\right)$ corresponding to a threshold $h \in B$ is a vector sequence whose $r$ th member equals to the threshold vector $x(r)_{(h)}$. Similarly, the threshold coordinate-orbit $\mathscr{O}_{i}\left(A_{(h)}, x_{(h)}\right)$ is the scalar sequence $\left(x_{i}(r)_{(h)} ; r \in \mathbb{N}_{0}\right)$.

For given $A \in B(n, n), x \in B(n)$, denote $H(A)=$ $\left\{a_{i j} ; i, j \in N\right\}$ and $H(A, x)=H(A) \cup\left\{x_{i} ; x_{i} \leq\right.$ $\max H(A) ; i \in N\}$.

Theorem 3.2. [1] For $A \in B(n, n), x \in B(n), O<h \in$ $B, r \in \mathbb{N}$ and $i, j \in N$, i) $\left(A^{r}\right)_{i j} \geq h$ if and only if there is a path in $\mathscr{G}\left(A_{(h)}\right)$ from $i$ to $j$ of length $r$,

ii) $\mathscr{O}_{i}(A, x)(r) \geq h$ if and only if there is a an orbit path in $\mathscr{G}\left(A_{(h)}, x_{(h)}\right)$ starting at $i$ of lenght $r$.

Lemma 3.1. [17] The decomposition of a matrix over $B$ to its threshold matrices has the following properties:

i) $A=\bigoplus_{h \in H}\left(h \otimes A_{(h)}\right)$ for any set $H$ such that $H(A) \subseteq$ $H \subseteq B$.

ii) For any two $\otimes$-compatible matrices $A$ and $B$, $(A \otimes B)_{(h)}=A_{(h)} \otimes B_{(h)}$ for any $h \in B$. Hence $A \otimes B=\bigoplus_{h \in H}\left(h \otimes A_{(h)} \otimes B_{(h)}\right)$ for any set $H$ such that $H(A) \cup H(B) \subseteq H \subseteq B$.

\subsection{Orbit stability}

In this part we shall deal with the so-called stable orbits. We give a necessary and sufficient condition for the stability of an orbit.

Definition 3.5. An orbit $\mathscr{O}(A, x)$ is stable if $\operatorname{per}(A, x)=1$.

Denote by $\mathscr{O}^{\text {per }}(A, x)$ and $\mathscr{O}_{i}^{\text {per }}(A, x)$ the periodic part of $\mathscr{O}(A, x)$ and $\mathscr{O}_{i}(A, x)$, respectively, i.e., $\mathscr{O}^{\text {per }}(A, x)=$ $(x(r) ; r>\operatorname{def}(A, x))$ and $\mathscr{O}_{i}^{\text {per }}(A, x)=\left(x_{i}(r) ; r>\right.$ $\operatorname{def}(A, x, i))$. By $(O)$ and $(I)$ we understand the infinite sequences of the same elements $O$ and $I$, respectively.

For a given $A \in B(n, n), x \in B(n)$ and $i \in N$ denote $h(A, x, i)=\max \left\{h \in H(A, x) ; \mathscr{O}_{i}^{\text {per }}\left(A_{(h)}, x_{(h)}\right) \neq(O)\right\}$.

Lemma 3.2. Let $A \in B(n, n)$ and $x \in B(n)$. An orbit $\mathscr{O}(A, x)$ is stable if and only if $\operatorname{per}\left(A_{(h(A, x, i))}, x_{(h(A, x, i))}, i\right)=1$ for each $i \in N$.

Proof. It follows from Lemma 3.1 i) that $x_{i}(r)=$ $\bigoplus_{h \in x_{i}} h \otimes \bigoplus_{(h)} h \otimes x_{i}(r)_{(h)}$ for each $r>$ $h \in H(A, x) \quad h \leq h(A, x, i)$ $\max _{\in \in H(A, x, i)} \operatorname{def}\left(A_{(h)}, x_{(h)}, i\right), \quad$ or equivalently, $\quad \mathscr{O}_{i}^{\text {per }}(A, x)=$ $\bigoplus_{h \leq h(A, x, i)} h \otimes \mathscr{O}_{i}^{\mathrm{per}}\left(A_{(h)}, x_{(h)}\right)$.

$h \leq h(A, x, i)$

If there exists $i \in N$ such that $\operatorname{per}\left(A_{(h(A, x, i))}, x_{(h(A, x, i))}, i\right) \neq$ 1 then $\mathscr{O}_{i}^{\text {per }}\left(A_{(h(A, x, i))}, x_{(h(A, x, i))}\right) \neq(I)$. Since an orbit is always periodic, we have $\operatorname{per}\left(A_{h(A, i)}, x_{h(A, i)}\right)=p>1$, which implies that there exist $k, l>\operatorname{def}(A, x, i)$, such that $x_{i}(k+m p)_{(h(A, x, i))}=I$ and $x_{i}(l+m p)_{(h(A, x, i))}=O$, and consequently $x_{i}(k+m p)=h(A, x, i)$ and $x_{i}(l+m p)<h(A, x, i)$, for each $m \in \mathbb{N}_{0}$. Then $\operatorname{per}(A, x, i) \neq 1$ and by Theorem 3.1 ii) we get $\operatorname{per}(A, x) \neq 1$. Thus $\mathscr{O}(A, x)$ is not stable.

Conversely, if $\operatorname{per}\left(A_{(h(A, x, i))}, x_{(h(A, x, i))}, i\right)=1$ for each $i \in N$ then for each $i \in N$ we get $x_{i}(r)=h(A, x, i)$ for each $r>\operatorname{def}(A, x, i)$, i.e., $\operatorname{per}(A, x, i)=1$. By Theorem 3.1 ii) we get $\operatorname{per}(A, x)=1$.

Remark 3.2. Gavalec in [3] has shown that the computation of the coordinate-orbit period is NP-hard, which is because the period of a matrix may be exponential large. In the following we shall deal with the class of circulant matrices, which have the period bounded by $n$. Thus the computation of the coordinate-orbit period becomes polynomial.

In [17], we can find the $O\left(n^{3} \log n\right)$ algorithm for computing the orbit period in general case. 


\subsection{Interval orbits}

Similarly to [2], [6], [11], [12] we define an interval matrix $\mathbf{A}$ and an interval vector $\mathbf{X}$.

Definition 3.6. Let $\underline{A}, \bar{A} \in B(n, n), \underline{A} \leq \bar{A}, \underline{x}, \bar{x} \in B(n), \underline{x} \leq$ $\bar{x}$. An interval matrix $\mathbf{A}$ and interval vector $\mathbf{X}$ with bounds $\underline{A}, \bar{A}$ and $\underline{x}, \bar{x}$, respectively, are defined as follows

$$
\begin{gathered}
\mathbf{A}=[\underline{A}, \bar{A}]=\{A \in B(n, n) ; \underline{A} \leq A \leq \bar{A}\}, \\
\mathbf{X}=[\underline{x}, \bar{x}]=\{x \in B(n) ; \underline{x} \leq x \leq \bar{x}\} .
\end{gathered}
$$

In like manner we can define the notion of an interval orbit.

Definition 3.7. Let $\mathbf{A}=[\underline{A}, \bar{A}]$ and $\mathbf{X}=[\underline{x}, \bar{x}]$. An interval orbit $\mathbf{O}(\mathbf{A}, \mathbf{X})$ is defined as follows:

$$
\mathbf{O}(\mathbf{A}, \mathbf{X})=\{\mathscr{O}(A, . x) ; A \in \mathbf{A}, x \in \mathbf{X}\} .
$$

\section{ORBITS OF CIRCULANT MATRICES}

In this section we shall deal with the special class of matrices, the circulant matrices. We prove the assertions needed in the next section.

Definition 4.1. A matrix $A \in B(n, n)$ is called circulant, if it has a form

$A=\left(\begin{array}{cccccc}a_{0} & a_{1} & a_{2} & \ldots & a_{n-2} & a_{n-1} \\ a_{n-1} & a_{0} & a_{1} & \ldots & a_{n-3} & a_{n-2} \\ \vdots & \vdots & \vdots & & \vdots & \vdots \\ a_{1} & a_{2} & a_{3} & \ldots & a_{n-1} & a_{0}\end{array}\right)$.

We denote a circulant matrix by $A\left(a_{0}, \ldots, a_{n-1}\right)$.

For a given circulant matrix $A=A\left(a_{0}, a_{1}, \ldots, a_{n}\right)$ let us denote by $h_{1}, h_{2}, \ldots, h_{r}$ the elements of $H(A)$ in the following way: $h_{1}>h_{2}>\cdots>h_{r}$. It is well known that the threshold digraphs $\mathscr{G}(A, h)$ for each $h \in H(A)$ consist from isomorphic strongly connected components. Moreover we can compute the vertex sets of these components in the following way (for details see [9]).

For each $j \in\{1,2, \ldots, r\}$ denote

$$
P^{j}=\left\{i \in N_{0} ; a_{i} \geq h_{j}\right\} .
$$

Then the number of strongly connected components of $\mathscr{G}\left(A_{\left(h_{j}\right)}\right)$ is

$m^{j}=\operatorname{gcd}\left(P^{j} \cup\{n\}\right)$

and we denote them by $\mathscr{K}_{1}^{j}, \mathscr{K}_{2}^{j}, \ldots, \mathscr{K}_{m^{j}}^{j}$. For each $j \in$ $\{1,2, \ldots, r\}, i \in\left\{1,2, \ldots, m^{j}\right\}$ the vertex set of $\mathscr{K}_{i}^{j}$ is given by the formula

$V_{i}^{j}=\left\{k \in N ; k \equiv i \bmod m^{j}\right\}$

The cardinality of the vertex set $V_{i}^{j}$ is $v_{j}=\frac{n}{m^{j}}$ for each $i \in\left\{1,2, \ldots, m^{j}\right\}$.

Remark 4.1. Let us note, that if $k, l$ are not lying in the same strongly connected component of $\mathscr{G}\left(A_{(h)}\right)$, then there is not edge from $k$ to $l$ in $\mathscr{G}\left(A_{(h)}\right)$.
Denote $I(A)=\left\{i \in N_{0} ; a_{i}=\max _{k \in N_{0}} a_{k}\right\} \cup\{n\}$. The following theorem gives the formula for the computation the period of a circulant matrix.

Theorem 4.1. [5] Let $A\left(a_{0}, \ldots, a_{n-1}\right)$ be a circulant matrix, $I(A)=\left\{n, i_{0}, i_{1}, \ldots, i_{k-1}\right\}$. Then

$$
\begin{gathered}
\operatorname{per}(A)=\operatorname{gcd}\left(\frac{n}{\operatorname{gcd}\left(n, i_{0}\right)}, \frac{i_{0}-i_{1}}{\operatorname{gcd}\left(i_{0}, i_{1}\right)}, \frac{i_{1}-i_{2}}{\operatorname{gcd}\left(i_{1}, i_{2}\right)}, \ldots\right. \\
\left.\frac{i_{k-1}-i_{k-2}}{\operatorname{gcd}\left(i_{k-2}, i_{k-1}\right)}\right) .
\end{gathered}
$$

According to [5] the computational complexity of (4) is $O(n)$.

Corollary 4.1. Let $A$ be a circulant matrix. Then $\operatorname{per}(A) \leq$ $n$.

The structure of the eigenspace of circulant matrices is described in [7].

Lemma 4.1. [17] Let $A \in B(n, n)$ and $x \in B(n)$ be binary. Then the periods of coordinate-orbits to the nodes of the same strongly connected component are equal.

Lemma 4.2. [17] Let $A \in B(n, n)$ and $x \in B(n)$ be binary. Let $\left(v_{0}, v_{1}, \ldots, v_{l}\right)$ be path in a non-trivial strongly connected component $\mathscr{K}$ of $\mathscr{G}(A)$. Then $\mathscr{O}_{v_{0}}(A, x)(r+l)=$ $\mathscr{O}_{v_{0}}(A, x)(r)$ for each $r \geq \operatorname{def}\left(A, x, v_{l}\right)$.

Corollary 4.2. Let $A \in B(n, n)$ and $x \in B(n)$ be binary and $\mathscr{K} \in \operatorname{SCC}^{\star}(A, x)$. If $i, j \in V_{\mathscr{K}}$ and $\mathscr{O}_{i}^{\text {per }}(A, x) \neq(O)$ then $\mathscr{O}_{j}^{\mathrm{per}}(A, x) \neq(O)$.

Definition 4.2. Let $A$ be a circulant matrix and $h \in$ $B$. We say that a strongly connected component $\mathscr{K} \in$ $\mathrm{SCC}^{\star} \mathscr{G}\left(A_{(h)}, x_{(h)}\right)$ has the property $(P)$, if for each $i \in V_{\mathscr{K}}$ there exists $r \in \mathbb{N}_{0}$ such that for each $k>r$ there exists an orbit path from $i$ in $\mathscr{K}$ of length $k$.

Theorem 4.2. Let $A\left(a_{0}, a_{1}, \ldots, a_{n-1}\right)$ be a circulant matrix and $x \in B(n)$. An orbit $\mathscr{O}(A, x)$ is stable if and only if for each $i \in N$ the property $(P)$ holds for the strongly connected component $\mathscr{G}\left(A_{(h(A, x, i))}, x_{(h(A, x, i))}\right)[i]$.

Proof. Denote $\mathscr{K}_{i}=\mathscr{G}\left(A_{(h(A, x, i))}, x_{(h(A, x, i))}\right)[i]$. If for each $i \in N$ the strongly connected component $\mathscr{K}_{i}$ has the property $(P)$ then $\operatorname{per}\left(A_{(h(A, x, i))}, x_{(h(A, x, i))}, i\right)=1$ for each $i \in N$ by Theorem 3.2 ii). In view of Lemma 3.2 we get $\operatorname{per}(A, x)=1$.

For the converse implication suppose that there exists $i \in N$ such that $\mathscr{K}_{i}$ does not have the property $(P)$. Then there exists $j \in V_{\mathscr{K}_{i}}$ such that for each $r \in \mathbb{N}$ there exists $k>r$ such that there is no orbit path of length $k$ from $j$ in $\mathscr{K}_{i}$. In view of Remark 4.1 there is no orbit path from $j$ to node which does not lie in $\mathscr{K}_{i}$. Thus $\mathscr{O}_{j}^{\text {per }}\left(A_{(h(A, x, i))}, x_{(h(A, x, i))}\right) \neq(I)$ and, according to Corollary 4.2 $\mathscr{O}_{j}^{\text {per }}\left(A_{(h(A, x, i))}, x_{(h(A, x, i))}\right) \neq(O)$ which implies $\operatorname{per}\left(A_{(h(A, x, i))}, x_{(h(A, x, i))}, j\right) \neq 1$. Then, by Lemma 4.1 $\operatorname{per}\left(A_{(h(A, x, i))}, x_{(h(A, x, i))}, i\right) \neq 1$. In view of Lemma 3.2 we get $\operatorname{per}(A, x) \neq 1$. 
Lemma 4.3. Let $A, C \in B(n, n)$ and $x, y \in B(n)$ be binary and $\mathscr{K}_{1} \in \mathrm{SCC}^{\star} \mathscr{G}(A, x), \mathscr{K}_{2} \in \mathrm{SCC}^{\star} \mathscr{G}(C, y)$ be such that $\mathscr{K}_{1} \subseteq \mathscr{K}_{2}, x_{i} \leq y_{i}$ for each $i \in V_{\mathscr{K}_{1}}$, and $\mathscr{K}_{1}$ have the property $(P)$. Then $\mathscr{K}_{2}$ has the property $(P)$.

Proof. Let $i \in V_{\mathscr{K}_{2}}$. If $i \in V_{\mathscr{K}_{1}}$ then the assertion trivially holds. If $i \in V_{\mathscr{K}_{2}} \backslash V_{\mathscr{K}_{1}}$ then there exists a path $\mathscr{P}$ from $i$ to $j$, where $j \in V_{\mathscr{K}_{1}}$. The existence of an orbit path from $j$ of length $k$ in $\mathscr{K}_{2}$ implies the existence of an orbit path from $i$ of length $k+|\mathscr{P}|$. If $r \in \mathbb{N}_{0}$ is such that for each $k>r$ there exists an orbit path from $j$ in $\mathscr{K}_{1}$ of length $k$, then there exists $s=r+|\mathscr{P}|$ such that for each $l>s$ there exists an orbit path from $i$ in $\mathscr{K}_{2}$ of length $l$. Thus $\mathscr{K}_{2}$ has the property $(P)$.

\section{INTERVAL CIRCULANT MATRICES}

The notions of an interval circulant matrix and a weak solvability of interval orbit of interval circulant matrix are defined in this section. A necessary and sufficient condition for the weak solvability of interval orbit of interval circulant matrix is proved. The notions of the possible and universal $\mathbf{X}$-robustness of an interval circulant matrix are defined and the polynomial algorithms for checking of them are introduced in this section.

Definition 5.1. An interval circulant matrix $\mathbf{A}^{C}$ is the set of all circulant matrices $A \in \mathbf{A}$ where

$\mathbf{A}=\left(\begin{array}{cccccc}\mathbf{a}_{0} & \mathbf{a}_{1} & \mathbf{a}_{2} & \ldots & \mathbf{a}_{n-2} & \mathbf{a}_{n-1} \\ \mathbf{a}_{n-1} & \mathbf{a}_{0} & \mathbf{a}_{1} & \ldots & \mathbf{a}_{n-3} & \mathbf{a}_{n-2} \\ \vdots & \vdots & \vdots & & \vdots & \vdots \\ \mathbf{a}_{1} & \mathbf{a}_{2} & \mathbf{a}_{3} & \ldots & \mathbf{a}_{n-1} & \mathbf{a}_{0}\end{array}\right)$,

and $\mathbf{a}_{i}=\left[\underline{a}_{i}, \bar{a}_{i}\right]$ for each $i \in N$. We denote an interval circulant matrix by $\mathbf{A}^{C}\left(\mathbf{a}_{0}, \ldots, \mathbf{a}_{n-1}\right)$.

There are matrices, that are not circulant, in $\mathbf{A}$, so $\mathbf{A} \neq \mathbf{A}^{C}$. On the other hand $\underline{A}, \bar{A} \in \mathbf{A}^{C}$, therefore the set $\mathbf{A}^{C}$ is always non-empty.

Definition 5.2. An interval orbit $\mathbf{O}\left(\mathbf{A}^{C}, \mathbf{X}\right)$ is weakly stable if there exist $A \in \mathbf{A}^{C}$ and $x \in \mathbf{X}$ such that $\mathscr{O}(A, x)$ is stable.

\subsection{Weak stability of interval orbits of interval circu- lant matrices}

Denote $\underline{a}=\max _{k \in N_{0}} \underline{a}_{k}$. Let us define the circulant matrix $A^{*}=A^{*}\left(a_{0}^{*}, a_{1}^{*}, \ldots, a_{n-1}^{*}\right)$ as follows:

$a_{i}^{*}=\min \left\{\underline{a}, \bar{a}_{i}\right\} \quad$ for each $i \in N_{0}$.

Lemma 5.1. [13| Let $x \in B(n)$ and $\mathbf{A}^{C}=\mathbf{A}^{C}\left(\mathbf{a}_{0}, \ldots, \mathbf{a}_{n-1}\right)$ be given. Then there exists a matrix $A \in \mathbf{A}^{C}$ such that $\operatorname{per}(A, x)=1$ if and only if $\operatorname{per}\left(A^{*}, x\right)=1$.

For $m \in \mathbb{N}$, denote $M=\{1,2, \ldots, m\}$. Let $\underline{y}, \bar{y} \in B(m)$, $\underline{y} \leq \bar{y}$. We denote $\mathbf{Y}=[\underline{y}, \bar{y}]=\{y \in B(m) ; \underline{y} \leq \bar{y} \leq \bar{y}\}$.

Denote $\underline{y}=\max _{k \in M} \underline{y}_{k}$. Let us define the vector $y^{*}=$ $\left(y_{1}^{*}, y_{2}^{*}, \ldots, y_{m}^{*}\right)$ as follows:

$y_{i}^{*}= \begin{cases}\underline{y}_{\underline{y}} & \text { if } \bar{y}_{i} \geq \underline{y}, \\ \underline{y}_{i} & \text { otherwise. }\end{cases}$ for each $i \in M$. For a given vector $y \in \mathbf{Y}$ let us denote $J(y)=\left\{i \in M ; y_{i}=\max _{j \in M} y_{j}\right\}$.

Lemma 5.2. Let $y^{*}$ be given by (6). Then $J(y) \subseteq J\left(y^{*}\right)$ for each $y \in \mathbf{Y}$.

Proof. If $J\left(y^{*}\right)=M$, then $J(y) \subseteq J\left(y^{*}\right)$ trivially holds. If $J\left(y^{*}\right)=\left\{i \in M ; \bar{y}_{i} \geq \underline{y}\right\} \neq M$ then for each $i \notin J\left(y^{*}\right)$ the inequality $\bar{y}_{i}<\underline{y}$ holds true. Consequently $\max _{i \neq J\left(y^{*}\right)} \bar{y}_{i}<\underline{y}$. Let $y \in \mathbf{Y}$ and $r \in J(y)$ be arbitrary. We get

$$
y_{r}=\max _{k \in M} y_{k} \geq \max _{k \in M} \underline{y}_{k}=\underline{y}>\max _{i \neq J\left(y^{*}\right)} \bar{y}_{i} \geq \max _{i \notin J\left(y^{*}\right)} y_{i}
$$

which implies $r \in J\left(y^{*}\right)$. Consequently $J(y) \subseteq J\left(y^{*}\right)$ for each $y \in \mathbf{Y}$.

For each $y \in B(m)$ denote $h(y)=\max _{i \in M} y_{i}$. From the previous the next lemma follows.

Lemma 5.3. For each $y \in \mathbf{Y}$ the inequality $y_{(h(y))} \leq y_{\left(h\left(y^{*}\right)\right)}^{*}$ is satisfied.

Proof. It follows directly from Lemma 5.2 .

For a given matrix $A\left(a_{0}, a_{1}, \ldots, a_{n-1}\right)$ and an interval vector $\mathbf{X}$ we define the vector $x(A)$ by the following algorithm.

In Algorithm $\mathscr{A}$, an auxiliary vector $p \in\{0,1\}^{n}$ will be used. The vector $p$ will be used to register the entries of $x(A)$ which are assigned a final value, i.e., $p_{k}=0$ till $x_{k}(A)$ is not assigned the final value.

Algorithm $\mathscr{A}$ : Computing the vector $x(A)$

Input: $A\left(a_{0}, a_{1}, \ldots, a_{n-1}\right)$, an interval vector $\mathbf{X}$

Output: the vector $x(A)$

begin

order the elements of $H(A)$ in such way that $h_{1}>h_{2}>$ $>\cdots>h_{r}$;

$x(A):=\underline{x} ; p_{k}:=0$ for each $k \in N$;

for $j=1: r$ do

if there exists $k \in N$ such that $p_{k}=0$ then

find $P^{j}$, compute $m^{j}$ by (2);

for $i=1: m^{j}$ do

compute $V_{i}^{j}$ by 3 ; ;

if $p_{k}=1$ for some $k \in V_{i}^{j}$ then

$p_{k}:=1$ for each $k \in V_{i}^{j}$;

endif

if $p_{k}=0$ for each $k \in V_{i}^{j}$ and $\max _{k \in V_{i}^{j}} x_{k} \geq h_{j}$ then

$p_{k}:=1$ for each $k \in V_{i}^{j}$;

if $j=1$ then $x_{k}(A):=\bar{x}_{k}$ for each $k \in V_{i}^{j}$;

endif

if $j>1$ then

$\underline{y}:=\left(\underline{x}_{i_{1}}, \underline{x}_{i_{2}}, \ldots, \underline{x}_{i_{v_{j}}}\right), \bar{y}:=\left(\bar{x}_{i_{1}}, \bar{x}_{i_{2}}, \ldots, \bar{x}_{i_{v_{j}}}\right)$, where $V_{i}^{j}=\left\{i_{1}, i_{2}, \ldots, i_{v_{j}}\right\}, i_{1}<i_{2}<\cdots<i_{v_{j}}$; 


\author{
compute $y^{*}$ by 6 ; \\ $x_{i_{k}}(A):=y_{k}^{*}$ for $k \in\left\{1,2, \ldots, v_{j}\right\}$; \\ endif \\ endif \\ enddo \\ endif \\ enddo
}

end

Remark 5.1. Let $A \in B(n, n)$ and $\mathbf{X}$ be given. Algorithm $\mathscr{A}$ computes the vector $x(A)$ in $O\left(n^{2}\right)$ arithmetic operations.

We illustrate Algorithm $\mathscr{A}$ in the following example.

Example 5.1. Compute the vector $x(A)$ if $\mathbf{X}=([9,11],[6,9],[8,10],[4,8],[8,9],[3,4],[8,8],[4,5])$ and $A=A(3,3,8,3,4,3,6,4)$.

First, we order the elements of $A$. We get $h_{1}=8, h_{2}=$ $6, h_{3}=4, h_{4}=3$. Initially we put $x(A):=x$ and $p_{k}:=0$ for $k \in\{1,2, \ldots, 8\}$.

For $j=1$ we have $P^{1}=\{2\}, m^{1}=\operatorname{gcd}(2,8)=2$.

For $i=1, V_{1}^{1}=\{1,3,5,7\}$. Since $p_{k}=0$ for each $k \in V_{1}^{1}$ and $\max _{k \in V_{1}^{1}} \underline{x}_{k}=9 \geq h_{1}$ we put $p_{k}:=1$ and $x_{k}(A):=\bar{x}_{k}$ for $k \in V_{1}^{1}$, i.e., $x_{1}(A):=11, x_{3}(A):=10, x_{5}(A):=9, x_{7}(A):=8$.

For $i=2, V_{2}^{1}=\{2,4,6,8\}$. Since $\max _{k \in V_{2}^{1}} x_{k}=6<h_{1}$, the entries $p_{k}$ and $x_{k}(A)$ for $k \in V_{2}^{1}$ will be not changed in this step.

For $j=2$ we have $P^{2}=\{2,6\}, m^{2}=\operatorname{gcd}(2,6,8)=2$. For $i=1, V_{1}^{2}=\{1,3,5,7\}$. Since $p_{k}=1$ for each $k \in V_{1}^{2}$, we will continue with $i=2$.

For $i=2, V_{2}^{2}=\{2,4,6,8\}$. Since $p_{k}=0$ for each $k \in V_{1}^{1}$ and $\max _{k \in V_{2}^{2}} x_{k}=6 \geq h_{2}$ we put $p_{k}:=1$ for $k \in V_{2}^{2}$. We compute $y^{*}=(6,6,3,4)^{T}$ and put $x_{2}(A):=6, x_{4}(A):=6, x_{6}(A):=$ $3, x_{8}(A):=4$.

For $j=3$ we have $p_{k}=1$ for each $k \in\{1,2, \ldots, 8\}$ the algorithm $\mathscr{A}$ ends with the output

$$
x(A)=(11,6,10,6,9,3,8,4)^{T} .
$$

Lemma 5.4. Let $A\left(a_{0}, a_{1}, \ldots, a_{n-1}\right)$ and $\mathbf{X}$ be given. There exists a vector $x \in \mathbf{X}$ such that $\operatorname{per}(A, x)=1$ if and only if $\operatorname{per}(A, x(A))=1$, where the vector $x(A)$ is given by Algorithm $\mathscr{A}$.

Proof. Suppose that $\operatorname{per}(A, x(A)) \neq 1$. We will prove that $\operatorname{per}(A, x) \neq 1$ for each $x \in \mathbf{X}$

Let $l \in N$ be such that $\mathscr{G}\left(A_{(h(A, x(A), l))}, x(A)_{(h(A, x(A), l))}\right)[l]$ does not have the property $(P)$. Let $j \in\{1,2, \ldots, r\}$ be the least number such that $\max x(A)_{k} \geq h_{j}$ where $i \in$ $k \in V_{i}^{j}$

$\left\{1,2, \ldots, m^{j}\right\}$ is such that $l \in V_{i}^{j}$. We shall distinguish two cases.

Case 1. If $j=1$ then for each $x \in \mathbf{X}$ there exists $k \in V_{i}^{j}$ such that $x_{k} \geq h_{1}$. Then $h(A, x(A), l)=$ $h(A, x, l)=h_{1}$ for each $x \in \mathbf{X}$. Since $x_{k}(A)=\bar{x}_{k} \geq x_{k}$ for each $k \in V_{i}^{j}$, we get $x_{k}(A)_{(h(A, x(A), l))}=x_{k}(A)_{\left(h_{1}\right)} \geq$
$x_{k_{\left(h_{1}\right)}}=x_{k_{(h(A, x, l))}}$ for each $x \in \mathbf{X}$ and for each $k \in V_{i}^{j}$. Since $\mathscr{G}\left(A_{h(A, x, l)}, x_{h(A, x, l)}\right)[l]=\mathscr{G}\left(A_{(h(A, x(A), l))}, x(A)_{(h(A, x(A), l))}\right)[l]$ and $\mathscr{G}\left(A_{(h(A, x(A), l))}, x(A)_{(h(A, x(A), l))}\right)[l]$ does not have property $(P)$, then for each $x \in \mathbf{X}$ the strongly connected component $\mathscr{G}\left(A_{h(A, x, l)}, x_{h(A, x, l)}\right)[l]$ does not have property $(P)$ by Lemma 4.3 . Consequently $\operatorname{per}(A, x) \neq 1$.

Case 2. If $j>1$ then $h(A, x(A), l)=h(A, x(A), i)=$ $\max x(A)_{k}$. Let $x \in \mathbf{X}$ be arbitrary but fixed. Let $k \in V_{i}^{j}$

us define the vectors $\underline{y}=\left(\underline{x}_{i_{1}}, \underline{x}_{i_{2}}, \ldots, \underline{x}_{i_{v_{j}}}\right)$ and $\bar{y}=$ $\left(\bar{x}_{i_{1}}, \bar{x}_{i_{2}}, \ldots, \bar{x}_{i_{v_{j}}}\right), \quad$ where $V_{i}^{j}=\left\{i_{1}, i_{2}, \ldots, i_{v_{j}}\right\}, \quad i_{1}<$ $i_{2}<\cdots<i_{v_{j}}$. According to Algorithm $\mathscr{A}$ and (6) the equality $y^{*}=\left(x(A)_{i_{1}}, x(A)_{i_{2}}, \ldots, x(A)_{i_{v_{j}}}\right)$ holds true. We have $h\left(y^{*}\right)=h(A, x(A), i)$ and $h(y) \geq$ $h\left(y^{*}\right)$ for each $y \in[\underline{y}, \bar{y}]$. Then there exists $u \in V_{i}^{j}$ such that $y_{u}=h(y)$ and $h\left(A, x, i_{u}\right)=\min \left\{h_{1}, y_{u}\right\} \geq$ $h(A, x(A), i) . \quad$ Consequently $\mathscr{G}\left(A_{h\left(A, x, i_{u}\right)}, x_{h\left(A, x, i_{u}\right)}\right)\left[i_{u}\right] \subseteq$ $\mathscr{G}\left(A_{(h(A, x(A), l))}, x(A)_{(h(A, x(A), l))}\right)[l]$.

Moreover, by Lemma 5.3 , the inequality $y_{(h(y))} \leq$ $y_{\left(h\left(y^{*}\right)\right)}^{*}$ holds true which implies $x_{k_{\left(h\left(A, x, i_{u}\right)\right)}} \leq$ $x_{k_{(h(A, x(A), l))}}$ for each $k \in V_{\mathscr{G}\left(A_{h\left(A, x, i_{u}\right)}, x_{h\left(A, x, i_{u}\right)}\right)\left[i_{u}\right]}$. Since $\mathscr{G}\left(A_{(h(A, x(A), l))}, x_{(h(A, x(A), l))}\right)[l]$ does not have the property $(P)$ then $\mathscr{G}\left(A_{h\left(A, x, i_{u}\right)}, x_{h\left(A, x, i_{u}\right)}\right)\left[i_{u}\right]$ does not have the property $(P)$ for each $x \in \mathbf{X}$ by Lemma 4.3 . Consequently $\operatorname{per}(A, x) \neq 1$.

The converse implication is trivial.

Theorem 5.1. An interval orbit $\mathbf{O}\left(\mathbf{A}^{C}, \mathbf{X}\right)$ is weakly stable if and only if $\operatorname{per}\left(A^{*}, x\left(A^{*}\right)\right)=1$, where $A^{*}$ is the matrix defined by (5).

Proof. If there exists $x \in \mathbf{X}$ and $A \in \mathbf{A}^{C}$ such that $\operatorname{per}(A, x)=1$ then, by Lemma 5.1 there exists $x \in \mathbf{X}$ such that $\operatorname{per}\left(A^{*}, x\right)=1$. In view of Lemma 5.4 we get $\operatorname{per}\left(A^{*}, x\left(A^{*}\right)\right)=1$, where the vector $x\left(A^{*}\right)$ is given by Algorithm $\mathscr{A}$ with the input matrix $A^{*}$.

The converse implication is trivial.

Theorem 5.2. Let $\mathbf{A}^{C}$ and $\mathbf{X}$ be given. There is an algorithm which decides whether the interval orbit $\mathbf{O}\left(\mathbf{A}^{C}, \mathbf{X}\right)$ is weakly stable in $O\left(n^{2} \log n\right)$ arithmetic operations.

Proof. The complexity of checking the weak stability of $\mathbf{O}\left(\mathbf{A}^{C}, \mathbf{X}\right)$ using Theorem 5.1 consists of $O(n)$ operations needed for computing the matrix $A^{*}$ by formula (6), $O\left(n^{2}\right)$ operations needed for computing of the vector $x\left(A^{*}\right)$ according to Algorithm $\mathscr{A}$ and $O\left(n^{2} \log n\right)$ operations, which are necessary for determination the orbit period of $\mathscr{O}\left(A^{*}, x\left(A^{*}\right)\right)$. Thus the complexity of the complete algorithm is $O(n)+O\left(n^{2}\right)+O\left(n^{2} \log n\right)=O\left(n^{2} \log n\right)$.

Example 5.2. Check the weak stability of the interval orbit $\mathbf{O}\left(\mathbf{A}^{C}, \mathbf{X}\right)$ where

$$
\mathbf{X}=([9,11],[6,9],[8,10],[4,8],[8,9],[3,4],[8,8],[4,5])
$$
and

$\mathbf{A}^{C}=\mathbf{A}^{C}([2,3],[1,3],[8,10],[1,3],[2,4],[1,3],[2,6],[4,4])$.

First, we compute the matrix $A^{*}$. Since $\max _{k \in N_{0}} \underline{a}_{k}=8$, we get $A^{*}=A^{*}(3,3,8,3,4,3,6,4)$ by $(5)$.

Further, we need to compute the vector $x\left(A^{*}\right)$. Whereas the matrix $A^{*}$ is equal to the matrix $A$ from Example 5.1 
and the interval vector $\mathbf{X}$ is the same as in Example 5.1, we have $x\left(A^{*}\right)=(11,6,10,6,9,3,8,4)^{T}$.

It remains to compute $\operatorname{per}\left(A^{*}, x\left(A^{*}\right)\right)$. We have the orbit $\mathscr{O}\left(A^{*}, x\left(A^{*}\right)\right)=\left((11,6,10,6,9,3,8,4)^{T},(8,6,8,6,8,6,8,6)^{T}\right.$, $\left.(8,6,8,6,8,6,8,6)^{T}, \ldots\right)$.

It is easy to see that $\operatorname{per}\left(A^{*}, x\left(A^{*}\right)\right)=1$. In view of Theorem 5.1 the given interval orbit is weakly stable.

\section{REFERENCES}

[1] CECHLÁROVÁ, K.: On the powers of matrices in bottleneck/fuzzy algebra, Linear Alg. Appl. 175 (1992), 63-73.

[2] FIEDLER, M. - NEDOMA, J. - RAMÍK, J. - ROHN, J. - ZIMMERMANN, K.: Linear Optimization Problems with Inexact Data, Springer-Verlag, Berlin 2006.

[3] GAVALEC, M.: Computing orbit period in fuzzy algebra, Discrete Applied Mathematics 100 (2000), 4965 .

[4] GAVALEC, M.: Periodicity of matrices and orbits in fuzzy algebra, Tatra Mountains Math. Publ. 6 (1995), 35-46.

[5] GAVALEC, M.: Periods of special fuzzy matrices, Tatra Mountains Math. Publ. 16 (1999), 47-60.

[6] GAVALEC, M. - PLAVKA, J.: Monotone interval eigenproblem in max-min algebra, Kybernetika 46 (3) (2010), 387-396.

[7] GAVALEC, M. - TOMÁŠKOVÁ, H.: Eigenspace of a circulant max-min matrix, Kybernetika 46 (3) (2010), 397-404.

[8] GAVALEC, M. - ZIMMERMANN, K.: Classification of solutions to systems of two-sided equations with interval coefficients, Inter. J. of Pure and Applied Math. 45 (2008), 533-542.

[9] MOLNÁROVÁ, M. - MYŠKOVÁ, H. - PLAVKA, J.: The robustness of interval fuzzy matrices, submitted to Lin. Algebra Appl.

[10] HORVATH, T. - VOJTÁŠ, P.: Induction of fuzzy and annotated logic programs, Inductive Logic Programming 4455 (2007) 260-274.
[11] MYŠKOVÁ, H.: Interval systems of max-separable linear equations, Linear Alg. Appl. 403 (2005), 263272.

'[12] MYŠKOVÁ, H.: Control solvability of interval systems of max-separable linear equations, Linear Alg. Appl. 416 (2006), 215-223.

[13] MYŠKOVÁ, H. - PLAVKA, J.: X-robustness of interval circulant matrices in fuzzy algebra, submitted to Linear Alg. and Appl.

[14] ROHN, J.: Systems of Linear Interval Equations, Lin. Algebra Appl. 126 (1989), 39-78.

[15] SANCHEZ, E.: Resolution of eigen fuzzy sets equations, Fuzzy Sets and Systems 1 (1978), 69-74.

[16] SANCHEZ, E.: Medical diagnosis Medical diagnosis and composite relations, In: M. M. Gupta, R. K. Ragade, R. R. Yager, Eds, Advances in Fuzzy Set Theory and Applications (North- Holland, Amsterdam- New York, 1979), 437-444.

[17] SEMANČÍKOVÁ, B.: Orbits in fuzzy algebra, Linear Alg. Appl. 414 (2006), 38-63.

[18] TERANO, T. - TSUKAMOTO, Y.: Failure diagnosis by using fuzzy logic, In: Proc. IEEE Conference on Decision Control (New Orleans, LA, 1977), 13901395.

[19] ZADEH, I. A.: Toward a theory of fuzzy systems, In: R. E. Kalman, N. DeClaris, Eds., Aspects of Network and Systems Theory (Hold, Rinehart and Winston, New York, 1971), 209-245.

Received July 30, 2012, accepted September 28, 2012

\section{BIOGRAPHY}

Helena Myšková was born on 14.1. 1964. She graduated (RNDr) with distinction at the Faculty of Science at P. J. Šafárik University in Košice in 1987. She defended her $\mathrm{PhD}$ in the field of discrete mathematics in 2009; her thesis title was "Solving of systems and interval systems of linear equations over the max-plus and max-min algebra". She is working as a tutor at the Department of Mathematics and Theoretical Informatics since 1995. Her scientific research is focusing on interval computations in extremal algebras. 